\title{
Influência do magnésio na absorção de manganês e zinco por raízes destacadas de soja(1)
}

\author{
Adônis Moreira $^{(2)}$, Eurípedes Malavolta ${ }^{(3)}$, Reges Heinrichs ${ }^{(4)}$ e Roberto Tetsuo Tanaka(5)
}

Resumo - O objetivo deste trabalho foi avaliar a influência do Mg na absorção do Zn e do Mn em quatro cultivares de soja. Utilizou-se a técnica de cinética de absorção por raízes destacadas. As raízes foram colocadas em frascos com as seguintes combinações: três doses de $\mathrm{Mg}\left(1,0,3,0 \mathrm{e} 6,0 \mathrm{mmol} \mathrm{L}^{-1}\right)$ x cinco doses de $\mathrm{Zn}$ ou $\mathrm{Mn}\left(0,5,1,0,2,0,5,0\right.$ e 10,0 $\left.\mathrm{mmol} \mathrm{L}^{-1}\right)$, com três repetições. Com pipetador, foi colocado $14,8 \mathrm{MBq}$ de radiozinco ou de radiomanganês. Após uma hora de exposição, as raízes foram lavadas e secadas em estufa; em seguida foram pesadas e determinou-se a radioatividade. Os valores foram convertidos em micromoles por grama de matéria seca. Foram estimadas as variáveis $\mathrm{K}_{\mathrm{m}}$ e $\mathrm{V}_{\max }$ pela equação de Lineweaver \& Burk. As cultivares apresentaram comportamentos distintos na absorção de $\mathrm{Mg}, \mathrm{Mn}$ e $\mathrm{Zn}$; a inibição do $\mathrm{Mg}$ sobre o $\mathrm{Zn}$ e o Mn na soja é do tipo não competitiva, e as variáveis $\mathrm{K}_{\mathrm{m}}$ e $\mathrm{V}_{\text {max }}$ comportaram-se de modo independente no processo de absorção.

Termos para indexação: Glycine max, nutriente, inibição, carência mineral.

\section{Magnesium influence on manganese and zinc uptake by excised roots of soybean}

\begin{abstract}
The objective of this work was to evaluate the influence of $\mathrm{Mg}$ in the absorption of $\mathrm{Mn}$ and $\mathrm{Zn}$ in four soybean cultivars. Excised roots were used in the assays of absorption kinetics. The roots were placed in solutions with the following combinations: three concentrations of $\mathrm{Mg}(1.0,3.0$ and $\left.6.0 \mathrm{mmol} \mathrm{L}^{-1}\right) \mathrm{x}$ five concentrations of $\mathrm{Zn}$ or $\mathrm{Mn}\left(0.5,1.0,2.0,5.0\right.$ and $\left.10.0 \mu \mathrm{mol} \mathrm{L} \mathrm{L}^{-1}\right)$, with three replicates. To each of these solutions $14.8 \mathrm{MBq}$ of radiozinc or radiomanganese was pipetted. After one hour of exposure, the roots were washed, oven-dried, weighted, and the absorbed radioactivity determined. The obtained values were converted to micromoles per gram of dry matter. The $\mathrm{K}_{\mathrm{m}}$ and $\mathrm{V}_{\max }$ estimated values were determined by the Lineweaver \& Burk transformation. The soybean cultivars showed an effect of non-competitive inhibition between $\mathrm{Mg}$ and $\mathrm{Zn}$ and between $\mathrm{Mg}$ and $\mathrm{Mn}$. The cultivars had distinct behavior in absorption of $\mathrm{Mg}, \mathrm{Mn}$ and $\mathrm{Zn}$. The $\mathrm{K}_{\mathrm{m}}$ and $\mathrm{V}_{\max }$ values were mutually independent in the absorption process of roots.
\end{abstract}

Index terms: Glycine max, nutrient, inhibition, mineral deficiency.

\section{Introdução}

No Brasil, grande parte da área cultivada com soja situa-se na região dos Cerrados cujos solos possu-

(1) Aceito para publicação em 13 de setembro de 2002.

Extraído da tese de doutorado apresentada pelo primeiro autor à Universidade de São Paulo (USP). Projeto financiado pela Fapesp.

(2) Embrapa-Centro de Pesquisa Agroflorestal da Amazônia Ocidental, Caixa Postal 319, CEP 69011-970 Manaus, AM. E-mail: adonis@cpaa.embrapa.br

(3) USP, Centro de Energia Nuclear na Agricultura (Cena), Caixa Postal 96, CEP 13400-970 Piracicaba, SP. Bolsista do CNPq. E-mail: mala@ cena.usp.br

(4) USP, Cena. Bolsista da Fapesp. E-mail: rheinric@ @ena.usp.br

(5) Instituto Agronômico, Caixa Postal 28, CEP 13001-970 Campinas, SP. E-mail: tanakart@cec.iac.br em baixa capacidade de troca catiônica, alta capacidade de adsorção de $\mathrm{P}$ e elevadas concentrações de Al e Mn trocáveis ou disponíveis (Lopes, 1984; Komatuda, 1988).

Até recentemente havia grande preocupação com a toxidez de alguns elementos, destacando-se o $\mathrm{Mn}$ (Franco \& Döbereiner, 1971; Pavan \& Bingham, 1981). Atualmente, porém, observam-se várias culturas com deficiência desse nutriente, entre as quais se destaca a soja (Novais et al., 1989; Abreu et al., 1994).

A deficiência de Mn tem se tornado comum em áreas de cultivo, sobretudo em solos de baixa fertilidade natural, chegando a causar, em algumas situações, redução no rendimento de grãos de até $50 \%$ (Tanaka et al., 1992). Nessas áreas, tal deficiência foi atribuída à aplicação de altas doses de calcário que elevaram o $\mathrm{pH}$ a valores próximos da neutralidade. 
A aplicação de grandes quantidades de corretivo pode causar um novo equilíbrio dos íons na solução do solo em detrimento dos micronutrientes (Lindsay, 1991) e, conseqüentemente, reduzir a absorção destes pelas plantas, a menos que o desbalanço seja compensado pelo uso de fertilizantes contendo esses nutrientes.

Sabe-se que em áreas de Cerrado é comum a deficiência de $\mathrm{Zn}$, ainda que o nutriente faça parte das formulações de adubos usados no plantio (Malavolta \& Moreira, 1999). A calagem pode induzir a deficiência de Zn (Rogers \& Wu, 1948) quando feita antes da semeadura da soja, ou pelo efeito residual, como na rotação com o trigo ou milheto. Tal deficiência é agravada com a utilização de calcário dolomítico, pois a maior quantidade de $\mathrm{Mg}$ aplicado inibe a absorção de $\mathrm{Zn}$, por se tratar de elementos com valência, raio iônico e grau de hidratação semelhantes (KabataPendias \& Pendias, 1984).

Assim, resultados de pesquisas sobre os mecanismos de absorção ativa de íons pelas plantas superiores ajustam-se com a hipótese da existência de carregadores iônicos de natureza enzimática, que têm número finito de sítios de ligação. Desse modo, a eficiência de absorção de um dado nutriente pode ser obtida por meio das variáveis cinéticas $\mathrm{K}_{\mathrm{m}} \mathrm{e} \mathrm{V}_{\max }$ da equação de Michaelis-Menten (Epstein, 1972; Marschner, 1995).

O presente trabalho foi baseado na hipótese de que as deficiências de $\mathrm{Mn}$ e de $\mathrm{Zn}$ podem ocorrer não somente pelo efeito da calagem, mas também pelo efeito inibidor do $\mathrm{Mg}$ contido no calcário e em fertilizantes.

O objetivo deste trabalho foi estudar o efeito do $\mathrm{Mg}$ na absorção de $\mathrm{Mn}$ e $\mathrm{Zn}$ por raízes destacadas de quatro cultivares de soja.

\section{Material e Métodos}

Os experimentos foram realizados no Centro de Energia Nuclear da Agricultura (USP-Cena, Piracicaba, SP). As sementes das cultivares IAC 17, FT Estrela, IAC 15-1 e DM Nobre foram colocadas para germinar em vermiculita umedecida com $\mathrm{CaSO}_{4} \cdot 2 \mathrm{H}_{2} \mathrm{O} 10^{-4} \mathrm{~mol} \mathrm{~L}^{-1}$. Após uma semana, as plântulas foram transferidas para bandejas com 40 L de solução de Johnson et al. (1957), diluída a 1/5, arejada constantemente, até o estádio V2 (Fehr et al., 1971). Posteriormente, foram transferidas para outra bandeja com a mesma solução, omitindo-se, conforme o experimento, Mg e Zn ou Mg e Mn, até o estádio V4 (Fehr et al., 1971). $\mathrm{O}$ volume da solução foi completado diariamente com água destilada e desionizada.

No estádio V4, as raízes foram cortadas pouco abaixo do caule e todo o sistema radicular foi acondicionado em frascos tipo "snap cap", com $100 \mathrm{~mL}$ de solução, em delineamento de blocos casualizados em esquema fatorial, com as seguintes combinações: três doses de $\mathrm{Mg}(1,0,3,0 \mathrm{e}$ 6,0 $\left.\mathrm{mmol} \mathrm{L}^{-1}\right) \mathrm{x}$ cinco doses de $\mathrm{Zn}$ ou $\mathrm{Mn}(0,5,1,0,2,0$, 5,0 e 10,0 $\mu \mathrm{mol} \mathrm{L}^{-1}$ ) (Schmid et al., 1965), com três repetições. Foi colocado, conforme o experimento, $14,8 \mathrm{MBq}$ de radiozinco (meia vida $=245$ dias) ou radiomanganês ( meia vida $=291$ dias), iniciando-se a aeração, que durou uma hora. As raízes foram lavadas em frascos com água destilada e desionizada, agitando-as durante um minuto, procedimento repetido três vezes (Noggle \& Fried, 1960). Posteriormente, foram secadas em estufa com circulação forçada a $65^{\circ} \mathrm{C}$ até atingir peso constante e, em seguida, foram pesadas e fez-se a contagem da atividade do ${ }^{65} \mathrm{Zn}$ ou do ${ }^{54} \mathrm{Mn}$, usando espectofotômetro gama monocanal acoplado a um cristal cintilador sólido de $\mathrm{NaI}$ (TI).

Os valores obtidos em contagem por minuto (cpm) foram convertidos em micromoles por grama de matéria seca por 60 minutos de absorção. Foram determinados o $\mathrm{K}_{\mathrm{m}} \mathrm{e} \mathrm{V}_{\text {max }}$ pelas transformações de Lineweaver \& Burk (1934), a partir da equação de Michaelis-Menten.

$\mathrm{v}=\frac{\mathrm{V}_{\max }[\mathrm{M}]}{[\mathrm{M}]+\mathrm{K}_{\mathrm{m}}}$

em que: v é a velocidade de absorção; $V_{\max }$ é a velocidade máxima de absorção; [M] é a concentração iônica externa; $\mathrm{K}_{\mathrm{m}}$ é a constante de Michaelis-Menten.

Tomando-se a recíproca dos dois membros da equação (1) obtém-se a transformação de Lineweaver \& Burk (1934):

$1 / \mathrm{v}=1 / \mathrm{V}_{\max }+\mathrm{K}_{\mathrm{m}} / \mathrm{V}_{\max } \cdot 1 /[\mathrm{M}]$.

Ajustou-se uma regressão linear, em que $\mathrm{x}=1 /[\mathrm{M}] \mathrm{e}$ $\mathrm{y}=1 / \mathrm{v}$ e os valores de $\mathrm{V}_{\max }$ e $\mathrm{K}_{\mathrm{m}}$ na equação $\mathrm{y}=\mathrm{a}+\mathrm{bx}$ foram estimados pelas fórmulas: $\mathrm{V}_{\max }=1 / \mathrm{a}$ e $\mathrm{K}_{\mathrm{m}}=\mathrm{b} / \mathrm{a}$.

Após as transformações dos dados, foram feitas análises de variância, teste $\mathrm{F}$ e, quando necessário, o teste de comparação de médias (Tukey 5\%) e regressões (Pimentel-Gomes, 1990).

\section{Resultados e Discussão}

A absorção do $\mathrm{Zn}$ por raízes destacadas aumentou com o incremento das doses desse nutriente na solução nutritiva, com diferenças significativas en- 
tre as cultivares (Tabela 1). No entanto, com o aumento na concentração de $\mathrm{Mg}$ de $1,0 \mathrm{mmol} \mathrm{L}^{-1}$ para 6,0 mmol L ${ }^{-1}$ na solução, dentro de cada dose de $\mathrm{Zn}$, houve diminuição, na média, de $63,5 \%$ na absorção de zinco. Tal resultado concorda com os obtidos por Sadana \& Takkar (1983), que, estudando o efeito do $\mathrm{Mg}$ na absorção e translocação de ${ }^{65} \mathrm{Zn}$, observaram que a adição de $20 \mathrm{mmol} \mathrm{L}^{-1}$ de $\mathrm{Mg}$ na solução reduz significativamente a absorção do $\mathrm{Zn}$ pelas raízes do arroz.

A redução na absorção de $\mathrm{Zn}$ pelas raízes, com o incremento na concentração de $\mathrm{Mg}$ na solução, foi quase a mesma nas cinco doses de zinco. Segundo Brar \& Sekhon (1976) e Sadana \& Takkar (1983), esse comportamento é característico de inibição do tipo não competitiva.

A inibição não competitiva foi também confirmada pela relação entre o $\mathrm{Zn}$ absorvido e a sua concentração na solução obtida na transformação de Lineweaver $\&$ Burk. Os coeficientes angulares e as ordenadas do ponto de intersecção mudaram com o incremento das doses de $\mathrm{Mg}$ de modo que as relações entre o coeficiente angular e o ponto de intersecção permaneceram quase constantes (Tabela 2).

Esses resultados indicam que na presença de altas concentrações de $\mathrm{Mg}$, a absorção de $\mathrm{Zn}$ é negativamente afetada, o que é agravado na maioria dos solos tropicais, tendo em vista ser este micronutriente um dos mais limitantes nesses solos (Lopes, 1984). Um dos procedimentos para minimizar tal efeito é aumen- tar as quantidades de $\mathrm{KCl}$ nas soluções; a presença do $\mathrm{Cl}$ na solução aumenta a absorção de $\mathrm{Zn}$ pelo efeito do íon acompanhante, além do efeito inibitório existente entre o Mg e o K (Malavolta et al., 1997).

Os valores de $\mathrm{K}_{\mathrm{m}}$ e $\mathrm{V}_{\max }$ foram influenciados pelos níveis de $\mathrm{Mg}$ e pelas cultivares (Tabela 2). As plantas sob a dose $1,0 \mathrm{mmol} \mathrm{L}^{-1} \mathrm{de} \mathrm{Mg}$ apresentaram, na média, valores de $\mathrm{K}_{\mathrm{m}} \mathrm{e}_{\max } 45,2 \%$ e $73,7 \%$, respectivamente maiores que o nível $6,0 \mathrm{mmol} \mathrm{L}^{-1} \mathrm{de}$ magnésio. Essas mudanças nos valores de $\mathrm{K}_{\mathrm{m}} \mathrm{e} \mathrm{V}_{\max }$ podem ser explicadas pelo aumento ou decréscimo do número de sítios de absorção por unidade de peso radicular (Karunaratne et al., 1986).

Os valores de $\mathrm{K}_{\mathrm{m}}$ e $\mathrm{V}_{\text {max }}$ apresentaram diferenças entre as cultivares, destacando-se os menores valores de $\mathrm{K}_{\mathrm{m}}$ nas cultivares IAC 15-1 e FT Estrela, nas concentrações 1,0 $\mathrm{mmol} \mathrm{L}^{-1}$ e 3,0 $\mathrm{mmol} \mathrm{L}^{-1}$ de $\mathrm{Mg}$ (Tabela 2). Na dose 6,0 mmol L-1 de $\mathrm{Mg}$, os menores valores de $\mathrm{K}_{\mathrm{m}}$ estimados ocorreram nas cultivares IAC 15-1 e DM Nobre. Esse resultado indica a maior afinidade íon-carregador dessas cultivares dentro de cada concentração de Mg na solução, levando, então, à maior eficiência na absorção de um dado elemento, neste caso o zinco. Na seleção de plantas ou cultivares eficientes na absorção de nutrientes, temse procurado plantas com baixos valores de $\mathrm{K}_{\mathrm{m}}$ (Nielsen \& Barber, 1978; Furlani, 1988; Stefanutti, 1997).

A constante $\mathrm{V}_{\max }$ diminuiu significativamente em todas as cultivares com o aumento das concentra-

Tabela 1. A absorção de ${ }^{65} \mathrm{Zn}$ por raízes destacadas de plantas de quatro cultivares de soja nas três concentrações de magnésio ${ }^{(1)}$.

\begin{tabular}{|c|c|c|c|c|c|c|c|c|c|c|c|}
\hline $\begin{array}{c}\mathrm{Zn} \\
\left(\mu \mathrm{mol} \mathrm{L}{ }^{-1}\right)\end{array}$ & $\begin{array}{c}\mathrm{Mg} \\
\left(\mathrm{mmol} \mathrm{L}^{-1}\right)\end{array}$ & IAC 15-1 & $\begin{array}{l}\text { IAC } 17 \\
---\left(\mu \mathrm{mol}^{6}\right.\end{array}$ & $\begin{array}{l}\text { FT Estrela } \\
\mathrm{Zn} / \mathrm{g} \text { de mat }\end{array}$ & $\begin{array}{l}\text { DM Nobre } \\
\text { téria seca)--- }\end{array}$ & Média & IAC 15-1 & $\begin{array}{l}\text { IAC } 17 \\
\text { (\% de red }\end{array}$ & $\begin{array}{l}\text { FT Estrela } \\
\text { 'ção na abso }\end{array}$ & $\begin{array}{l}\text { DM Nobre } \\
\text { ção de Zn). }\end{array}$ & Média \\
\hline \multirow[t]{3}{*}{0,5} & 1,0 & $0,725 \mathrm{a}$ & $0,755 \mathrm{a}$ & $0,676 a$ & $0,460 \mathrm{a}$ & 0,654 & - & - & - & - & - \\
\hline & 3,0 & $0,463 \mathrm{a}$ & $0,465 \mathrm{a}$ & $0,373 \mathrm{a}$ & $0,320 \mathrm{a}$ & 0,405 & 37,93 & 38,41 & 44,82 & 30,43 & 38,07 \\
\hline & 6,0 & $0,315 \mathrm{a}$ & $0,342 \mathrm{a}$ & $0,219 \mathrm{a}$ & $0,281 \mathrm{a}$ & 0,289 & 56,55 & 54,70 & 67,60 & 38,91 & 55,81 \\
\hline \multirow[t]{3}{*}{1,0} & 1,0 & $1,308 \mathrm{ab}$ & $1,486 \mathrm{ab}$ & $2,077 \mathrm{a}$ & $0,764 b$ & 1,409 & - & - & - & - & - \\
\hline & 3,0 & $0,708 \mathrm{a}$ & $0,930 \mathrm{a}$ & $0,548 \mathrm{a}$ & $0,657 \mathrm{a}$ & 0,711 & 45,87 & 37,41 & 73,61 & 14,00 & 49,54 \\
\hline & 6,0 & $0,540 \mathrm{a}$ & $0,593 \mathrm{a}$ & $0,306 \mathrm{a}$ & $0,396 \mathrm{a}$ & 0,450 & 58,71 & 60,09 & 85,27 & 48,17 & 68,06 \\
\hline \multirow[t]{3}{*}{2,0} & 1,0 & $2,322 \mathrm{ab}$ & $3,006 \mathrm{ab}$ & $4,424 \mathrm{a}$ & $1,720 \mathrm{~b}$ & 3,118 & - & - & - & - & - \\
\hline & 3,0 & $1,380 \mathrm{a}$ & $1,166 \mathrm{a}$ & $1,127 \mathrm{a}$ & $0,906 a$ & 1,145 & 40,57 & 61,21 & 74,52 & 47,32 & 63,28 \\
\hline & 6,0 & $0,869 \mathrm{a}$ & $1,009 \mathrm{a}$ & $0,770 \mathrm{a}$ & $0,828 \mathrm{a}$ & 0,869 & 62,57 & 66,43 & 82,59 & 51,86 & 72,13 \\
\hline \multirow[t]{3}{*}{5,0} & 1,0 & $5,213 \mathrm{a}$ & $5,756 \mathrm{a}$ & $5,458 \mathrm{a}$ & $3,181 \mathrm{a}$ & 4,902 & - & - & - & - & - \\
\hline & 3,0 & $3,293 \mathrm{ab}$ & $3,895 \mathrm{a}$ & $2,612 b c$ & $2,174 \mathrm{c}$ & 2,994 & 36,83 & 32,33 & 52,14 & 31,66 & 38,92 \\
\hline & 6,0 & $2,214 \mathrm{a}$ & $2,085 \mathrm{a}$ & $1,698 \mathrm{a}$ & $1,464 \mathrm{a}$ & 1,865 & 57,53 & 63,78 & 68,89 & 53,98 & 61,95 \\
\hline \multirow[t]{3}{*}{10,0} & 1,0 & $8,829 \mathrm{ab}$ & $9,472 \mathrm{a}$ & $8,544 b$ & $4,975 \mathrm{c}$ & 7,955 & - & - & - & - & - \\
\hline & 3,0 & $5,782 \mathrm{~b}$ & $7,664 a$ & $5,505 b$ & $3,644 \mathrm{c}$ & 5,649 & 34,51 & 19,09 & 35,57 & 26,75 & 28,99 \\
\hline & 6,0 & $3,460 \mathrm{ab}$ & $3,925 \mathrm{a}$ & $2,833 \mathrm{~b}$ & $2,713 b$ & 3,233 & 60,81 & 58,56 & 66,84 & 45,47 & 59,36 \\
\hline
\end{tabular}

${ }^{(1)}$ Médias seguidas por letras distintas na mesma linha diferem entre si a $1 \%$ de probabilidade pelo teste de Tukey. 
ções de Mg (Tabela 2). Os valores de $\mathrm{V}_{\max }$ estimados foram menores nas cultivares DM Nobre e FT Estrela nas concentrações $1,0 \mathrm{mmol} \mathrm{L}^{-1} \mathrm{e}$ 3,0 $\mu \mathrm{mol} \mathrm{L}^{-1}$ de $\mathrm{Mg}$ e nas cultivares IAC 15-1 e DM Nobre na dose $6,0 \mu \mathrm{mol} \mathrm{L}^{-1}$ de $\mathrm{Mg}$, o que indica que essas cultivares, em determinada concentração de $\mathrm{Mg}$, possuem menor quantidade de sítios de absorção do carregador (Sacramento et al., 1999). Furlani (1988) e Stefanutti (1997), em estudos com genótipos de arroz, observaram que plantas mais adaptadas a elevadas fertilidades são aquelas que possuem $\mathrm{V}_{\max }$ correspondente aos valores mais altos.

Tabela 2. Equação de regressão, coeficiente de determinação e as variáveis $\mathrm{K}_{\mathrm{m}}\left(\mu \mathrm{mol} \mathrm{mL} \mathrm{mL}^{-1}\right) \mathrm{e}_{\max }\left(\mu \mathrm{mol} \mathrm{g}{ }^{-1} \mathrm{MS}^{-1}\right)$ determinados mediante as equações de Lineweaver \& Burk (1934), em relação a absorção de Zn por raízes destacadas durante 60 minutos.

\begin{tabular}{|c|c|c|c|c|}
\hline \multirow{2}{*}{$\begin{array}{c}\text { Magnésio } \\
\left(\mathrm{mmol} \mathrm{L}^{-1}\right)\end{array}$} & \multirow[t]{2}{*}{ Cultivares } & \multicolumn{3}{|l|}{ Zinco } \\
\hline & & Equação & $\mathrm{K}_{\mathrm{m}}$ & $\mathrm{V}_{\max }$ \\
\hline \multirow{4}{*}{$\overline{1,0}$} & IAC $15-1$ & $\mathrm{Y}=0,072+0,663 * * \mathrm{X} 0,99$ & 9,21 & 13,89 \\
\hline & IAC 17 & $\mathrm{Y}=0,033+0,643 * * \quad \mathrm{X} \quad 0,99$ & 19,49 & 30,03 \\
\hline & FT Estrela & $\mathrm{Y}=0,101+0,689 * * \mathrm{X} \quad 0,94$ & 6,82 & 9,90 \\
\hline & DM Nobre & $\mathrm{Y}=0,114+1,058 * * \mathrm{X} 0,99$ & 9,32 & 8,81 \\
\hline \multirow[t]{4}{*}{3,0} & IAC $15-1$ & $\mathrm{Y}=0,152+1,055 * * \mathrm{X} 0,97$ & 6,94 & 6,58 \\
\hline & IAC 17 & $\mathrm{Y}=0,115+1,026 * * \mathrm{X} 0,97$ & 8,92 & 8,69 \\
\hline & FT Estrela & $\mathrm{Y}=0,185+1,325 * * \mathrm{X} \quad 0,96$ & 7,16 & 5,41 \\
\hline & DM Nobre & $\mathrm{Y}=0,189+1,457 * * \mathrm{X} 0,98$ & 7,70 & 5,28 \\
\hline \multirow[t]{4}{*}{6,0} & IAC $15-1$ & $\mathrm{Y}=0,254+1,522 * * \mathrm{X} 0,98$ & 5,99 & 3,94 \\
\hline & IAC 17 & $\mathrm{Y}=0,215+1,384 * \mathrm{X}^{2} 0,99$ & 6,44 & 4,65 \\
\hline & FT Estrela & $\mathrm{Y}=0,189+1,457 * * \mathrm{X} 0,98$ & 7,71 & 5,29 \\
\hline & DM Nobre & $\mathrm{Y}=0,383+1,691 * * \mathrm{X} \quad 0,96$ & 4,42 & 2,61 \\
\hline
\end{tabular}

**Significativo pelo teste $\mathrm{F}$ a $1 \%$ de probabilidade.
As relações existentes entre a quantidade de $\mathrm{Zn}$ absorvida ( $\mu \mathrm{mol}{ }^{65} \mathrm{Zn} / \mathrm{g}$ de matéria seca de raiz) e a quantidade de $\mathrm{Zn}$ na solução $\left(\mu \mathrm{mol} \mathrm{L}{ }^{-1}\right)$ mostraram que a cultivar FT Estrela foi a mais eficiente e DM Nobre a menos eficiente na absorção de Zn nas três concentrações de Mg; as cultivares IAC 15-1 e IAC 17 apresentaram relações intermediárias. Segundo Sacramento et al. (1999), essa relação é indicativo da eficiência dos mecanismos de absorção, podendo-se considerar mais eficiente a cultivar capaz de acumular maior quantidade de $\mathrm{Zn}$ em seus tecidos radiculares, a partir da concentração externa do nutriente na solução.

Nielsen \& Barber (1978), trabalhando com plantas inteiras de milho, encontraram relação direta entre plantas com alta $V_{\max }$ e absorção de nutrientes. Os resultados obtidos por esses autores, associados aos do presente trabalho, permitem deduzir que a determinação dos valores da $\mathrm{V}_{\max }$ seja mais um componente na avaliação da absorção de $\mathrm{Mg}$ e zinco.

A absorção de Mn por raízes destacadas decresceu com o aumento da concentração de $\mathrm{Mg}$ (Tabela 3). O efeito depressivo das doses de $\mathrm{Mg}$ foi similar em cada dose de Mn, com diferenças, em alguns tratamentos, somente entre cultivares. Segundo Foy et al. (1969) e Stefanutti (1997), as diferenças de comportamento das plantas na absorção dos nutrientes são controladas, principalmente, por fatores genéticos.

Tabela 3. Absorção de ${ }^{54} \mathrm{Mn}$ por raízes destacadas de plantas de quatro cultivares de soja nas três concentrações de magnésio $^{(1)}$.

\begin{tabular}{|c|c|c|c|c|c|c|c|c|c|c|c|}
\hline $\mathrm{Mn}$ & $\mathrm{Mg}$ & IAC $15-1$ & IAC 17 & FT Estrela & DM Nobre & Média & IAC $15-1$ & IAC 17 & FT Estrela & DM Nobre & Média \\
\hline$\left(\mu \mathrm{mol} \mathrm{L}{ }^{-1}\right)$ & $\left.(\mathrm{mmol} \mathrm{L})^{-1}\right)$ & - & $-\left(\mu \mathrm{mol}^{54}\right.$ & $\mathrm{Mn} / \mathrm{g}$ de maté & ́ria seca) ----- & & & $(\%$ de rec & ção na absorç & ão de $\mathrm{Mn}$ ) -- & \\
\hline \multirow[t]{3}{*}{0,5} & 1,0 & $0,219 \mathrm{a}$ & $0,113 a$ & $0,063 b$ & $0,069 b$ & 0,116 & - & - & - & - & 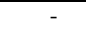 \\
\hline & 3,0 & $0,148 \mathrm{a}$ & $0,056 \mathrm{~b}$ & $0,037 b$ & $0,048 b$ & 0,072 & 32,42 & 50,44 & 41,27 & 30,43 & 37,93 \\
\hline & 6,0 & $0,074 \mathrm{a}$ & $0,044 \mathrm{a}$ & $0,020 \mathrm{a}$ & $0,026 \mathrm{a}$ & 0,041 & 66,21 & 61,06 & 68,25 & 62,31 & 64,65 \\
\hline \multirow[t]{3}{*}{1,0} & 1,0 & $0,352 \mathrm{a}$ & $0,161 \mathrm{~b}$ & $0,121 b$ & $0,137 b$ & 0,192 & - & - & - & - & - \\
\hline & 3,0 & $0,269 \mathrm{a}$ & $0,088 \mathrm{~b}$ & $0,060 \mathrm{~b}$ & $0,091 \mathrm{~b}$ & 0,127 & 23,58 & 45,34 & 50,41 & 33,58 & 33,85 \\
\hline & 6,0 & $0,164 \mathrm{a}$ & $0,078 \mathrm{~b}$ & $0,041 \mathrm{~b}$ & $0,049 \mathrm{~b}$ & 0,083 & 53,40 & 51,55 & 66,12 & 64,23 & 56,77 \\
\hline \multirow[t]{3}{*}{2,0} & 1,0 & $0,567 \mathrm{a}$ & $0,359 \mathrm{~b}$ & $0,220 \mathrm{c}$ & $0,260 \mathrm{c}$ & 0,352 & - & - & - & - & - \\
\hline & 3,0 & $0,487 \mathrm{a}$ & $0,171 \mathrm{~b}$ & $0,167 b$ & $0,134 b$ & 0,240 & 14,11 & 52,37 & 24,09 & 48,46 & 31,82 \\
\hline & 6,0 & $0,252 \mathrm{a}$ & $0,153 \mathrm{~b}$ & $0,079 \mathrm{c}$ & $0,075 \mathrm{c}$ & 0,140 & 55,56 & 57,38 & 64,09 & 71,15 & 60,23 \\
\hline \multirow[t]{3}{*}{5,0} & 1,0 & $1,061 \mathrm{a}$ & $0,734 \mathrm{~b}$ & $0,529 \mathrm{c}$ & $0,734 b$ & 0,765 & - & - & - & - & - \\
\hline & 3,0 & $0,883 \mathrm{a}$ & $0,452 b$ & $0,292 \mathrm{~d}$ & $0,348 \mathrm{c}$ & 0,495 & 16,78 & 38,42 & 44,80 & 52,59 & 35,29 \\
\hline & 6,0 & $0,633 \mathrm{a}$ & $0,362 \mathrm{~b}$ & $0,182 \mathrm{c}$ & $0,171 \mathrm{c}$ & 0,337 & 40,34 & 50,68 & 65,60 & 76,70 & 55,95 \\
\hline \multirow[t]{3}{*}{10,0} & 1,0 & $1,382 b$ & $1,488 \mathrm{a}$ & $0,856 \mathrm{~d}$ & $1,297 \mathrm{c}$ & 1,256 & - & - & - & - & - \\
\hline & 3,0 & $1,239 \mathrm{a}$ & $0,777 \mathrm{~b}$ & $0,549 \mathrm{c}$ & $0,766 \mathrm{~b}$ & 0,833 & 10,35 & 47,78 & 35,86 & 40,94 & 33,68 \\
\hline & 6,0 & $0,720 \mathrm{a}$ & $0,521 \mathrm{~b}$ & $0,311 \mathrm{c}$ & $0,254 \mathrm{c}$ & 0,452 & 47,90 & 64,99 & 63,67 & 80,42 & 64,01 \\
\hline
\end{tabular}

${ }^{(1)}$ Médias seguidas por letras distintas na mesma linha diferem entre si a $1 \%$ de probabilidade pelo teste de Tukey. 
$\mathrm{O}$ incremento na concentração de $\mathrm{Mg}$ de $1,0 \mathrm{mmol} \mathrm{L}^{-1}$ para 3,0 e 6,0 mmol L-1 diminuiu progressivamente a absorção de Mn (Tabela 3), com redução média de $34,5 \%\left(3,0 \mathrm{mmol} \mathrm{L}^{-1}\right)$ e $60,3 \%$ $\left(6,0 \mathrm{mmol} \mathrm{L}^{-1}\right)$. Esse resultado é evidência da interação que ocorre entre os nutrientes, com inibição na absorção de Mn e Zn, pela maior presença de Mg na solução (Kabata-Pendias \& Pendias, 1984).

A redução na absorção de Mn corrobora os resultados de Mass et al. (1969), que, ao estudarem a influência do Mg na absorção de Mn em cevada, observaram que a inibição entre o $\mathrm{Mg}$ e Mn é do tipo não competitiva (Tabela 4). Nesse tipo de inibição, o ponto de intersecção nas ordenadas e o coeficiente angular mudam com a presença do inibidor (Epstein, 1972; Malavolta et al., 1997).

A inibição não competitiva é ocasionada pela deformação do sítio não ativo do carregador, de modo que não ocorre a formação do complexo na velocidade usual e, uma vez formado, o complexo não se desdobra na velocidade normal para originar os produtos (Lehninger et al., 1995). Nesse tipo de inibição, a maior absorção de um elemento não pode ser anulada pelo aumento da concentração do outro, o que não ocorre na inibição competitiva, em que o inibidor I combina-se com o mesmo sítio ativo do carregador R; neste caso, a inibição pode ser desfeita aumentando-se a concentração do nutriente (Hopkins, 1995; Malavolta et al., 1997).

Tabela 4. Equação de regressão, coeficiente de determinação e as variáveis $\mathrm{K}_{\mathrm{m}}\left(\mu \mathrm{mol} \mathrm{mL} \mathrm{m}^{-1}\right) \mathrm{e} \mathrm{V}_{\max }\left(\mu \mathrm{mol} \mathrm{g}{ }^{-1} \mathrm{MS}^{-1}\right)$ determinados mediante as equações de Lineweaver \& Burk (1934), em relação a absorção de Mn por raízes destacadas durante 60 minutos.

\begin{tabular}{|c|c|c|c|c|c|}
\hline \multirow{2}{*}{$\begin{array}{c}\text { Magnésio } \\
\left(\mathrm{mmol} \mathrm{L}^{-1}\right)\end{array}$} & \multirow[t]{2}{*}{ Cultivares } & \multicolumn{4}{|c|}{ Manganês } \\
\hline & & Equação & $\mathrm{R}^{2}$ & $\mathrm{~K}_{\mathrm{m}}$ & $\mathrm{V}_{\max }$ \\
\hline \multirow[t]{4}{*}{1,0} & IAC $15-1$ & $\mathrm{Y}=0,739+2,108 * * \mathrm{X}$ & 0,91 & 2,85 & 1,35 \\
\hline & IAC 17 & $\mathrm{Y}=0,657+4,367 * * \mathrm{X}$ & 0,96 & 6,65 & 1,52 \\
\hline & FT Estrela & $\mathrm{Y}=0,516+7,618 * * \mathrm{X}$ & 0,99 & 14,76 & 1,94 \\
\hline & DM Nobre & $\mathrm{Y}=0,601+7,202 * * \mathrm{X}$ & 0,99 & 11,98 & 1,66 \\
\hline \multirow[t]{4}{*}{3,0} & IAC $15-1$ & $\mathrm{Y}=0,602+4,566 * * \mathrm{X}$ & 0,97 & 7,58 & 1,66 \\
\hline & IAC 17 & $\mathrm{Y}=0,995+8,883 * * \mathrm{X}$ & 0,98 & 8,93 & 1,01 \\
\hline & FT Estrela & $\mathrm{Y}=0,599+13,678^{* *} \mathrm{X}$ & 0,98 & 22,85 & 1,67 \\
\hline & DM Nobre & $\mathrm{Y}=1,145+9,860 * * \mathrm{X}$ & 0,98 & 8,61 & 0,87 \\
\hline \multirow[t]{4}{*}{6,0} & IAC $15-1$ & $\mathrm{Y}=0,954+6,891 * * \mathrm{X}$ & 0,98 & 7,22 & 1,05 \\
\hline & IAC 17 & $\mathrm{Y}=2,982+23,954 * * \mathrm{X}$ & 0,98 & 8,03 & 0,34 \\
\hline & FT Estrela & $\mathrm{Y}=2,492+15,270^{* *} \mathrm{X}$ & 0,99 & 6,13 & 0,40 \\
\hline & DM Nobre & $\mathrm{Y}=3,198+21,153^{* *} \mathrm{X}$ & 0,99 & 6,61 & 0,31 \\
\hline
\end{tabular}

** Significativo pelo teste $\mathrm{F}$ a $1 \%$ de probabilidade.
A constatação do tipo de inibição não competitiva demonstra que em solos com alta concentração de Mn, a aplicação de calcário pode diminuir o efeito de toxidez deste micronutriente em decorrência do aumento do $\mathrm{pH}$ do solo e da presença dos cátions $\mathrm{Ca}^{2+} \mathrm{e} \mathrm{Mg}^{2+}$, porém, em condições de carência limiar, a calagem pode agravar essa situação. Cabe salientar que ao contrário do que ocorre na inibição competitiva, em que, o aumento da concentração do nutriente pode desfazer a inibição, na não competitiva, conforme no presente trabalho, faz-se necessário o uso de outro meio para diminuir o efeito depressivo ocasionado pelo aumento da concentração de um determinado elemento, como a adição de íons acompanhantes, como $\mathrm{NO}_{3}{ }^{-}, \mathrm{Cl}^{-}$e $\mathrm{SO}_{4}{ }^{2-}$ (Malavolta et al., 1997).

Houve diminuição significativa na concentração de $\mathrm{Mn}$ nas raízes com o aumento de $\mathrm{Mg}$ na solução (Tabela 3). A cultivar IAC 15-1 absorveu a maior quantidade de $\mathrm{Mn}$ nas três concentrações de $\mathrm{Mg}$; o inverso foi obtido com a FT Estrela. As cultivares DM Nobre e IAC 17 tiveram comportamentos intermediários. A cultivar DM Nobre foi a única que apresentou diminuição significativa $(44,8 \%)$ no $\mathrm{K}_{\mathrm{m}}$ com o aumento da dose de $1,0 \mathrm{mmol} \mathrm{L}^{-1}$ para $6,0 \mathrm{mmol} \mathrm{L}^{-1}$ de $\mathrm{Mg}$ na solução (Tabela 4).

Os valores de $\mathrm{V}_{\max }$ estimados tiveram maior efeito na taxa de absorção de manganês. As cultivares IAC 17 e DM Nobre mostraram os menores valores de $\mathrm{V}_{\text {max }}$ nas concentrações 3,0 e 6,0 $\mathrm{mmol} \mathrm{L}^{-1}$ de $\mathrm{Mg}$, ao passo que na concentração $1,0 \mathrm{mmol} \mathrm{L}^{-1}$ de $\mathrm{Mg}$ os menores valores ficaram por conta de IAC $17 \mathrm{e}$ IAC 15-1, o que indica menor número de carregadores nas raízes destas cultivares.

Embora tenha havido diminuição no valor estimado de $\mathrm{V}_{\max }$, houve redução mais acentuada nos valores de $\mathrm{K}_{\mathrm{m}}$ estimados, resultando numa maior absorção de Mn pela DM Nobre (Tabela 3). De acordo com Vilela \& Anghinoni (1984), trabalhando com outras cultivares de soja, as mudanças em $\mathrm{V}_{\max }$ têm maior efeito na taxa de absorção de nutrientes do que $\mathrm{K}_{\mathrm{m}}$.

As relações existentes entre a quantidade de $\mathrm{Mn}$ absorvida ( $\mu \mathrm{mol}{ }^{54} \mathrm{Mn} / \mathrm{g}$ de matéria seca de raiz) e a quantidade de $\mathrm{Mn}$ na solução ( $\mu \mathrm{mol} \mathrm{L} \mathrm{L}^{-1}$ ) mostram que a cultivar IAC 15-1 foi a mais eficiente em acumular maior quantidade de Mn em seus tecidos radiculares e FT Estrela a menos eficiente; as cultivares DM Nobre e IAC 17 apresentaram relações intermediárias. 


\section{Conclusões}

1. Na soja, a inibição do Mg sobre a absorção do Zn e do Mn por raízes destacadas é do tipo não competitiva.

2. As cultivares apresentam comportamento distinto na absorção de nutrientes; independentemente da dose de $\mathrm{Mg}$, a cultivar FT Estrela tem maior capacidade de absorver Zn, e IAC 15-1, de absorver manganês.

3. Na soja, as variáveis $K_{m} e V_{\text {max }}$ estimadas comportam-se de modo independente no processo de absorção.

\section{Referências}

ABREU, C. A.; RAIJ, B.; TANAKA, R. T. Comportamento de cultivares de soja em solo deficiente em manganês. Revista Brasileira de Ciência do Solo, Campinas, v. 19 , n. 1, p. 149-152, 1994.

BRAR, M. S.; SEKHON, G. S. Effect on manganese on zinc- 65 absorption by rice seedlings and its translocation within the plants. Plant and Soil, Dordrecht, v. 44, p. $459-462,1976$.

EPSTEIN, E. Mineral nutrition of plants: principles and perspectives. New York: J. Wiley, 1972. 412 p.

FEHR, W. R.; CAVINESS, C. E.; BURMOOD, D. T.; PENNINGTON, J. S. Stage of development description for soybeans (Glycine $\max$ (L.) Merrill). Crop Science, Madison, v. 11, n. 6, p. 929-931, 1971.

FOY, C. D.; FLEMING, A. L.; ARMIGER, W. H. Differential tolerance of cotton varieties to excess manganese. Agronomy Journal, Madison, v. 61, n. 2, p. 690-694, 1969.

FRANCO, A. A.; DÖBEREINER, J. Toxidez de manganês de um solo ácido na simbiose soja - Rhizobium. Pesquisa Agropecuária Brasileira, Série Agronomia, Rio de Janeiro, v. 6, n. 4, p. 57-66, 1971.

FURLANI, A. M. C. Variações em parâmetros de cinética de absorção de fósforo de três linhagens de arroz. Revista Brasileira de Ciência do Solo, Campinas, v. 12, n. 1, p. 77-80, 1988.

HOPKINS, W. G. Plants and inorganic nutrients. In: HOPKINS, W. G. (Ed.). Introduction to plant physiology. Toronto: J. Wiley, 1995. p. 65-80.
JOHNSON, C. M.; STOUT, P. R.; BROYER, T. C.; CARLTON, A. B. Comparative chlorine requirements of different plant species. Plant and Soil, Dordrecht, v. 8, n. 1, p. 337-353, 1957.

KABATA-PENDIAS, A.; PENDIAS, H. Trace elements in soils and plants. Boca Raton: CRC Press, 1984. 315 p.

KARUNARATNE, R. S.; BAKER, J. H.; BARKER, A. V. Phosphorus uptake by mycorrhizal and non-mycorrhizal roots of soybeans. Journal of Plant Nutrition, New York, v. 9, p. 1303-1313, 1986.

KOMATUDA, C. R. N. Comportamento de variedades de soja (Glycine max (L.) Merrill) em condições de deficiência ou excesso de manganês em solução nutritiva. 1988. 62 f. Dissertação (Mestrado em Solos e Nutrição de Plantas) - Universidade Federal de Viçosa, Viçosa, 1988.

LEHNINGER, A. L.; NELSON, D. L.; COX, M. M. Princípios de bioquímica. São Paulo: Sarvier, 1995. 839 p.

LINDSAY, W. L. Inorganic equilibria affecting micronutrients in soils. In: MORTVEDT, J. J.; COX, F. R.; SHUMAN, L. M.; WELCH, R. M. (Ed.). Micronutrients in agriculture. Madison: American Society of Agronomy, 1991. p. 89-112.

LINEWEAVER, H.; BURK, D. The determination of enzyme dissociation constants. Journal of the American Chemical Society, Washington, v. 56, n. 1, p. 658-666, 1934.

LOPES, A. S. Solos sob "cerrado": características, propriedades e manejo. Piracicaba: Potafos, 1984. 162 p.

MAlavolta, E.; MOREIRA, A. Absorção de radiozinco por duas variedades de arroz. Piracicaba: Centro de Energia Nuclear na Agricultura, 1999. 5 p.

MALAVOLTA, E.; VITTI, G. C.; OLIVEIRA, S. A. Avaliação do estado nutricional de plantas: princípios e aplicações. Piracicaba: Potafos, 1997. 319 p.

MARSCHNER, H. Mineral nutrition of higher plants. San Diego: Academic, 1995. 889 p.

MASS, E. V.; MOORE, D. P.; MASON, B. J. Influence of calcium and magnesium on manganese absorption. Plant Physiology, Rockville, v. 44, p. 796-800, 1969.

NIELSEN, N. E.; BARBER, S. A. Differences among genotypes of corn in the kinetics of P uptake. Agronomy Journal, Madison, v. 70, n. 5, p. 695-698, 1978. 
NOGGLE, J. C.; FRIED, M. A kinetic analysis of phosphate absorption by excised roots of millet, barley and alfalfa. Soil Science Society of America Proceeding, Madison, v. 24, n. 1, p. 33-35, 1960.

NOVAIS, R. F.; NEVES, J. C. L.; BARROS, N. F.; SEDYAMA, T. Deficiência de Mn em plantas de soja cultivadas em solos de cerrado. Revista Brasileira de Ciência do Solo, Campinas, v. 13, n. 1, p. 199-204, 1989.

PAVAN, M. A.; BINGHAM, F. T. Toxidez de metais em plantas - I: caracterização de toxidez de manganês em cafeeiros. Pesquisa Agropecuária Brasileira, Brasília, v. 16 , n. 6 , p. 815-821, 1981.

PIMENTEL-GOMES, F. Curso de estatística experimental. Piracicaba: Nobel, 1990. 468 p.

ROGERS, L. H.; WU, C. Zinc uptake by oats as influenced by application of lime and phosphate. Journal of the American Society of Agronomy, Madison, v. 40, n. 2, p. $563-566,1948$.

SACRAMENTO, L. V. S.; MARTINEZ, H. E. P.; MONNERAT, P. H.; OLIVEIRA, L. M. Absorção de magnésio por raízes destacadas de cultivares de tomateiro. Scientia Agricola, Piracicaba, v. 56, n. 3, p. 509-515, 1999.
SADANA, U. S.; TAKKAR, P. N. Effect of calcium and magnesium on ${ }^{65}$ Zinc absorption and translocation in rice seedlings. Journal of Plant Nutrition, New York, v. 6, p. $705-715,1983$.

SCHMID, W. E.; HAAG, H. P.; EPSTEIN, E. Absorption of zinc by excised barley roots. Physiologia Plantarum, Copenhagen, v. 18, p. 860-869, 1965.

STEFANUTTI, R. Eficiência da absorção e utilização de fósforo e zinco por variedades de arroz. 1997. $93 \mathrm{f}$. Tese (Doutorado em Energia Nuclear na Agricultura) Universidade de São Paulo, Centro de Energia Nuclear na Agricultura, Piracicaba, 1997.

TANAKA, R. T.; MASCARENHAS, H. A. A.; BULISANI, E. A. Deficiência de manganês em soja induzida por excesso de calcário. Pesquisa Agropecuária Brasileira, Brasília, v. 27, n. 2, p. 247-250, 1992.

VILELA, L.; ANGHINONI, I. Morfologia do sistema radicular e cinética de absorção de fósforo em cultivares de soja afetados pela interação alumínio-fósforo. Revista Brasileira de Ciência do Solo, Campinas, v. 8, n. 1, p. 91-96, 1984. 